\title{
Testicular Myeloid Sarcoma
}

National Cancer Institute

\section{Source}

National Cancer Institute. Testicular Myeloid Sarcoma. NCI Thesaurus. Code C162469.

A rare myeloid sarcoma that arises from the testis. At presentation the involvement is usually unilateral. 